\title{
Métodos Algorítmicos para la optimización de rutas en el Sistema del Transporte Urbano
}

Campos-Vásquez Neicer, Maestro en Ciencias Económicas, Cueva-Clemente Carlos, Bautista-Zuñiga Lucia Maribel, Maestra en Administración Estratégica de Empresas, Sotomayor-Burga Juan Luis, Maestro en Ciencias Empresariales

Universidad Privada del Norte, Perú, neicer.campos@upn.edu.pe, N001655888@upn.pe, lucia.bautista@upn.edu.pe,

juan.sotomayor@upn.edu.pe

\begin{abstract}
RESUMEN - Este artículo muestra y determina los distintos métodos algorítmicos que fueron aplicados para la optimización de las rutas del sistema del transporté urbano a nivel nacional (Perú) $e$ internacional, debido al factor principal que es el crecimiento de la demanda(usuarios) y la poca respuesta de la oferta(rutas de transporte). El objetivo de este artículo es dar a conocer los diferentes enfoques y escenarios a partir de la aplicación de los algoritmos como : genéticos (evolutivo, simple) dijkstra, PSO, culturales(ACs), colonia de hormigas(ACO) y Tabú. Este análisis cualitativo y cuantitativo se realizó a través del método de recolección de datos de las distintas fuentes académicas como: Redalyc, Scopus, Scielo y el Motor de búsqueda de Google académico. Los resultados nos muestras las distintas formas de como poder optimizar una ruta establecida y la creación de una nueva ruta en el sistema de transporte urbano.
\end{abstract}

PALABRAS CLAVES: algoritmo genético, dijkstra, PSO, culturales (ACs) y colonia de hormigas.

ABSTRACT - This article shows and determines the different algorithmic methods that were applied to optimize the routes of the urban transport system at a national (Peru) and international level, due to the main factor that is the growth of demand (users) and the little response The objective of this article is to present the different approaches and scenarios from the application of algorithms such as: genetic (evolutionary, simple) dijkstra, PSO, cultural (ACs), colony of ants (ACO) and Taboo. This qualitative and quantitative analysis was carried out through the method of collecting data from different academic sources such as: Redalyc, Scopus, Scielo and the academic Google search engine. The results show us the different ways to optimize an established route and the creation of a new route in the urban transport system.

KEY WORDS: genetic algorithm, dijkstra, PSO, cultural (ACS) and ant colony.

\section{INTRODUCCIÓN}

Un algoritmo se puede conceptualizar como una serie de indicaciones que representan un modelo de solución para definido tipo de inconvenientes. O bien como un grupo de normas que llevadas a cabo en orden conducen a obtener la solución de un problema. Asimismo, los Algoritmos De los genes son procedimientos adaptativos, principalmente utilizados en inconvenientes de averiguación y optimización de parámetros, basados en la reproducción sexual y en el inicio de supervivencia del más apto [1].

En la actualidad debido al avance tecnológico hablar de algoritmos y su aplicabilidad no es nuevo, en sus diferentes tipos y aplicaciones, puesto que están siendo aplicados en todas las ramas de la ciencia y la vida cotidiana, en negocios, en medicina, etc [2]. Es por tal, que no es ajeno en el trasporte y más aún en estos días donde la innovación es el foco de actualidad.

En el año 1930 se propuso el problema del agente viajero, que consistía en obtener la ruta óptima para que un viajero pasara por todos los puntos de vista de un mapa, en otras palabras, extensamente estudiado a partir de entonces, y su estudio y resolución para hallar la ruta óptima sigue vigente actualmente. Al principio la resolución se realizaba con algoritmos de fuerza bruta, no obstante, dichos algoritmos se volvieron obsoletos gracias a la dificultad que se iba sumando al problema, por lo cual después surgieron resoluciones con algoritmos aproximados [3].

En 1979 Christoph E. Mandl planteó el problema de la red suiza de transporte y expuso una solución del problema. La representación del problema es una red de 15 nodos y 21 arcos, (ver figura 1).

Esta situación de análisis ha sido investigada por otros estudiosos como Baaj and Mahmassani entre otros, todos ellos obteniendo una mejora de rutas para el problema propuesto. Por consiguiente, descubrir resoluciones a optimizar y/o mejorar el transporte es un inconveniente del presente.
Digital Object Identifier (DOI):

http://dx.doi.org/10.18687/LEIRD2021.1.1.32

ISSN: 2414-6390 ISBN: 978-958-52071-9-6 


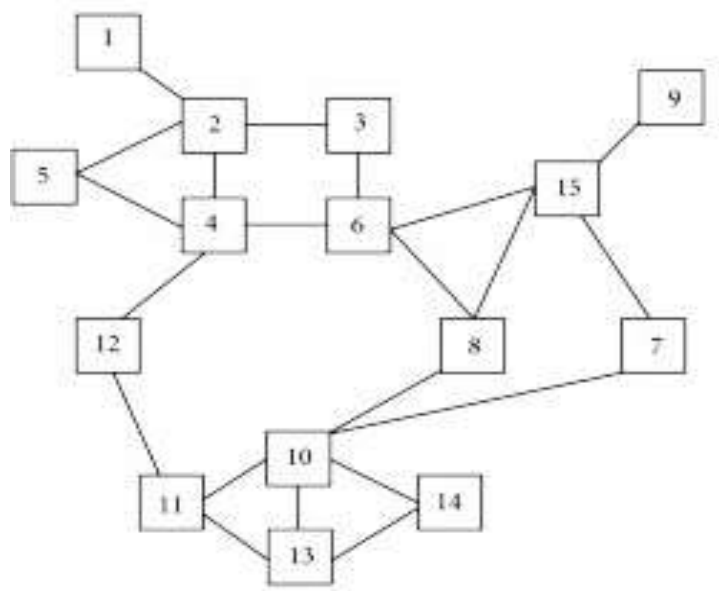

Figura 1: Representación Problema de Mandl [3].

La rápida urbanización en Lima ha llevado a un aumento de la población limeña por la migración de las personas del campo a la ciudad (Imagen $\mathrm{N}^{\circ} 1$ ) [4], es así como proporcionalmente se ha incrementado los servicios de movilidad, así mismo esto ha conllevado a una presión en donde se ha venido generando soluciones en la infraestructura del sistema del transporte urbano [5]. Este incremento implica nuevas oportunidades y desafíos para explorar la estructura y la dinámica del sistema de transporte en donde se busca optimizar el servicio del mismo.

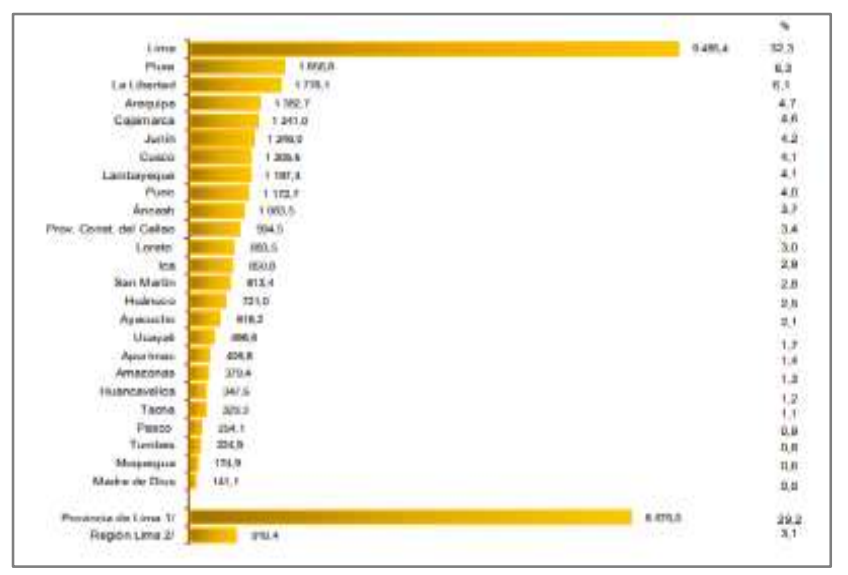

Gráfico $N^{o}$ 1: Población censada, según departamento 2017 (miles)

(INEI, 2017)

En esta misma línea [6] nos dice que Lima tiene inconvenientes serios en lo cual tiene relación con la circulación generalmente y en el transporte público en especial. Este caso perjudica en especial a los sectores de menores recursos y produce fuertes externalidades negativas. El sector metropolitano de Lima muestra problemas estructurales en la organización de su transporte y tránsito: el servicio de transporte público es deficiente, el tránsito es caótico, los tiempos de viaje son altos, existe un elevado índice de accidentes y una contaminación significativa generada por el parque automotor.
Desafortunadamente, con fuerte inquietud tenemos la posibilidad de mencionar que Lima y Callao, en este instante, ya se hallan en estado de colapso [7]. Es por tal que el transporte público representa un papel importante en el desarrollo de la vida de los pobladores de Lima, ya que se constituye el primordial modo de transporte de la localidad para transportar a la mayoría de la población para hacer sus ocupaciones cotidianas [8].

En el sistema de transporte tenemos desde el uso del GPS (sistema de posicionamiento global), hasta lo que es hoy la realidad aumentada, en análisis de ciencias de datos, entre otros, los cuales han influido positivamente es este rubro.

Actualmente el servicio de transporte de lima está conformado por el tren eléctrico, el sistema metropolitano, corredores complementarios, ruta de transporte público en autobús [9], pese a estar supuestamente estructurada no cubre la demanda existente. El sistema de transporte publico consta de cinco fases que tiene como objetivo brindar un servicio eficiente: Primero definir la ruta de servicio, definir la frecuencia de los buses, establecer la hora de llegada de las unidades a los paraderos, asignar unidades a cada ruta basados en frecuencia y horarios y asignar choferes a las unidades [10].

Los algoritmos son utilizados para resolver cualquier tipo de problemas en cualquier escenario, de tal manera que aplicarlo para optimizar el transporte de manera eficiente y eficaz nos llevara a tener resultados óptimos. En consecuencia se pretende investigar ¿Cuáles son los métodos o tipos de algoritmos utilizados y/o aplicados a la optimización de transporte urbano?, y como objetivo general se propuso conocer los distintos métodos que se han utilizado para la optimización de rutas de transporte aplicando algoritmos a través de treinta artículos de estudio encontrados en las distintas base de datos, lo cual nos ayudara a dar alternativas e ideas nuevas a la sociedad para implementar algoritmos en las operaciones de transporte y lograr optimizarlas en la ciudad de Lima.

\section{METODOLOGIA}

Esta recopilación de información se dio a través de las distintas fuentes académicas siguiendo una metodología de revisión sistemática. La pregunta de investigación para direccionar el tema fue la siguiente ¿Cuáles son los métodos o tipos de algoritmos utilizados y/o aplicados a la optimización de transporte urbano?

Una revisión sistemática ayuda a dar sentido a muchos tipos de datos, existen una manera de revisar todos los datos de una investigación sobre una cuestión concreta de forma sistemática y estandarizada [11]. Una revisión sistemática ayuda a dar una visión global objetiva y transparente de todas las pruebas que rodean a una pregunta concreta.

Para detallar el proceso de búsqueda información se utilizó buscadores académicos como de REDALYC, SCIELO, 
SCOPUS Y GOOGLE ACADEMICO en donde se obtuvo distintos artículos científicos, abordando temas como algoritmos genéticos, optimización del transporte, rutas y frecuencias, método de colonia de hormigas, el agente viajero, algoritmos heurísticos, metaheurística entre otros.

Dentro de nuestros criterios de búsqueda se consideró artículos con 12 años de antigüedad, así mismo se hizo una búsqueda exhaustiva de artículos internacionales en español e inglés con el fin de tener una visión más amplia en materia de investigación, según las palabras claves como "algoritmos", "métodos algorítmicos en el transporte urbano", "optimización de rutas con algoritmos en el transporte urbano".

Asimismo, se aplicó los criterios de selección y exclusión de los artículos científicos donde se analizaron información del periodo 2008-2020 en idiomas en inglés y español, descartando artículos que no se encontraban dentro del rango establecido, en cuanto al contenido de los artículos se excluyó a los que tenían temas similares o por duplicidad, este contenido se analizó por título de investigación, objetivo, resumen y año de publicación.

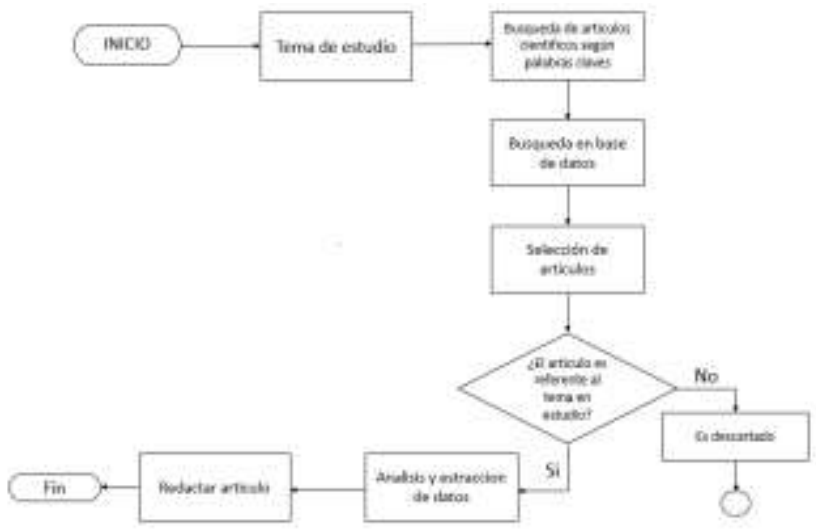

Figura $N^{o} 1$ : Diagrama de flujo de proceso de búsqueda de información Fuente: Elaboración propia

Para la construcción de la matriz de recolección de datos se consideró los siguientes aspectos: Numero de orden, origen, año, autor, titulo, motivo de exclusión, motivo de inclusión y descripción como lo muestra la (tabla $\mathrm{N}^{\mathrm{a}}$ 1).

\begin{tabular}{|c|c|c|c|c|c|c|}
\hline $\begin{array}{l}\mathrm{N}^{\text {o de }} \text { orden } \\
\text { Ordigen }\end{array}$ & Año & Autor & Titulo $\begin{array}{c}\text { Motivo } \\
\text { de } \\
\text { exclusión }\end{array}$ & $\begin{array}{c}\text { Motivo } \\
\text { de } \\
\text { inclusión }\end{array}$ \\
Tabla $N^{\circ} 1:$ Matriz de recolección de datos \\
Fuente: Elaboración propia
\end{tabular}

\section{RESULTADOS}

Como resultado de búsqueda y análisis de los artículos mencionados con relación a nuestra pregunta de investigación
¿Cuáles son los métodos o tipos de algoritmos utilizados y/o aplicados a la optimización de transporte urbano? Hemos obtenido en total 30 artículos (adjunto tabla $\mathrm{N}^{\mathrm{a}}$ 2), los cuales están distribuidos de la siguiente manera Redalyc, 12 artículos; Google académico, 8 artículos, Scopus, 1 artículo y Scielo, 9 artículos, dando una sumatoria de un total de 30 artículos.

\begin{tabular}{lll}
$\mathrm{N}^{\mathrm{o}}$ & BADE DE DATOS & CANTIDAD \\
\hline 1 & REDALYC & 12 \\
& GOOGLE & \\
2 & ACADEMICO & 8 \\
3 & SCOPUS & 1 \\
4 & SCIELO & 9 \\
\hline & TOTAL & 30
\end{tabular}

Tabla $N^{a} 2$ : Resumen de búsqueda

Elaboración propia

De acuerdo a lo anterior señalado se observa que de los artículos analizados y buscadores utilizados se encontró mayor información en Redalyc con $40 \%$, en Google académico con $27 \%$, en Scopus con 3\% y en Scielo 30\%, siendo Redalyc el buscador con más información relevante con nuestro tema de investigación tal como lo muestra el siguiente (Gráfico $\mathrm{N}^{\circ} 2$.)

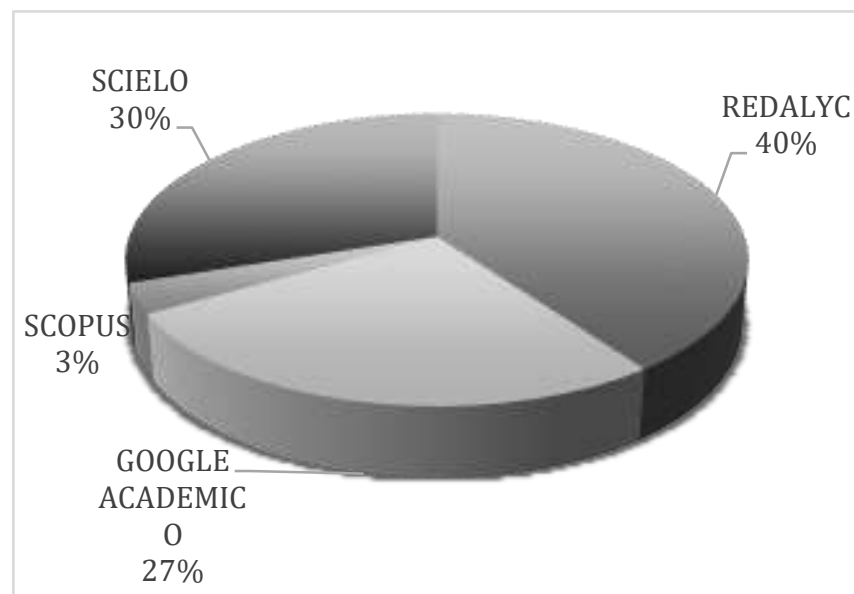

ఐREDALYC $\square$ GOOGLE ACADEMICO $\square$ SCOPUS $₫$ SCIELO

Gráfico $N^{o} 2$ : Total de participación de buscadores

Fuente: Elaboración propia

Asimismo, se obtuvo artículos de distintos continentes como América latina, Asia y Europa, siendo estos malasia 1 artículo, Chile 2 artículos, China 1 articulo, Uruguay 5 artículos, Colombia 4 artículos, cuba 1 articulo, Brasil 1 articulo, España 5 artículos, México 3 artículos, argentina 3 artículos y Perú 5 artículos como lo muestra el Grafico $\mathrm{N}^{\mathrm{a}} 3$. 


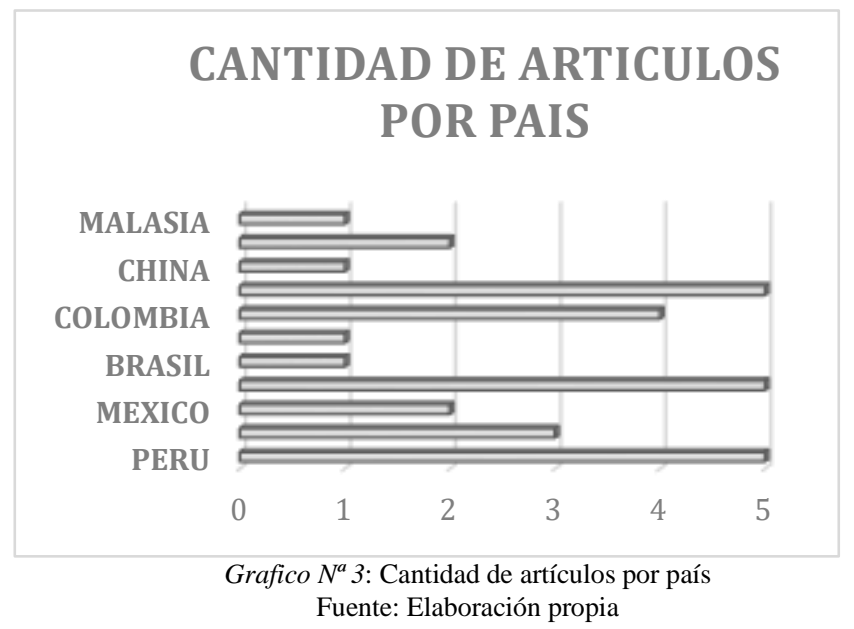

Como ya hemos explicado anteriormente en esta investigación se han analizado 30 artículos de los cuales han sido seleccionados 21 artículos que nos brindan información relevante y que responden a nuestra pregunta de investigación, objetivos principales y año de publicación, siendo estos distribuidos de la siguiente manera; 2008 dos publicaciones, 2011 dos publicaciones, 2012 una publicación, 2013 cinco publicaciones, 2014 dos publicaciones, 2015 dos publicaciones, 2016 dos publicaciones, 2017 dos publicaciones, 2018 una publicación, 2019 una publicación y 2020 una publicación como lo muestra la (Tabla $\mathrm{N}^{\mathrm{o}} 3$ ).

Los artículos analizados nos muestran un panorama en donde podemos ver respecto a la problemática señalada a inicios de este artículo, es decir, se observa que en gran parte de América Latina se han realizado investigaciones en busca de una solución con respecto al uso de algoritmos en el transporte urbano, asimismo podemos ver que en Europa también está latente la problemática mencionada, en consecuencia, podemos observar que este problema es de interés global.

En conclusión, este análisis de investigación nos permite señalar que existen distintos tipos y métodos algorítmicos aplicados para la optimización de las rutas en el sistema de transporte urbano el cual puede ser aplicado en nuestro país.

Tabla No 3:

Matriz de Recojo de Información

\begin{tabular}{|c|c|c|c|c|}
\hline $\mathbf{N}^{0}$ & Año & País & Autor & Titulo \\
\hline 01 & Perú & 2011 & $\begin{array}{l}\text { Luzmila pro-concepción, augusto } \\
\text { Cortez Vásquez, Walter contreras, } \\
\text { lázaro mota Alva }\end{array}$ & $\begin{array}{l}\text { Análisis y diseño de redes viales de transporte urbano } \\
\text { usando algoritmos genéticos: caso lima metropolitana }\end{array}$ \\
\hline 02 & México & 2013 & Claudia rodríguez Lemus & $\begin{array}{l}\text { Aplicación del algoritmo de colonia de hormigas para la } \\
\text { optimización del transporte público }\end{array}$ \\
\hline 03 & Perú & 2015 & Walter Alonso Barrantes ríos & $\begin{array}{l}\text { Implementación de un algoritmo recocido simulado para } \\
\text { el diseño de rutas de transporte público para lima centro }\end{array}$ \\
\hline 04 & Perú & 2017 & José miguel, sato Yamada & $\begin{array}{l}\text { Implementación de algoritmos meta heurísticos para la } \\
\text { definición de frecuencias y horarios para rutas de } \\
\text { transporte público }\end{array}$ \\
\hline 05 & España & 2013 & $\begin{array}{l}\text { Andrés aguado Aranda, } \\
\text { Javier Jiménez de vega }\end{array}$ & Optimización de rutas de transporte \\
\hline 06 & España & 2016 & $\begin{array}{l}\text { Samir Kanaan izquierdo } \\
\text { Carles ventura royo }\end{array}$ & $\begin{array}{l}\text { Optimización de rutas de transporte público con } \\
\text { algoritmos genéticos }\end{array}$ \\
\hline 07 & Colombia & 2019 & $\begin{array}{l}\text { Laura Betancourt delgado, } \\
\text { miguel a. becerra, }\end{array}$ & $\begin{array}{l}\text { Optimización del transporte público urbano mediante } \\
\text { algoritmos de búsqueda tabú y pso: Medellín, Colombia }\end{array}$ \\
\hline 08 & Chile & 2013 & $\begin{array}{l}\text { J. enrique Fernández 1., Joaquín de cea } \\
\text { ch. }\end{array}$ & $\begin{array}{l}\text { Una metodología para el diseño topológico de } \\
\text { sistemas de transporte público urbano de pasajeros }\end{array}$ \\
\hline 09 & Argentina & 2014 & $\begin{array}{l}\text { Ana c. olivera; Jessica a. carballido; } \\
\text { mariano frutos; Nélida b. brignole }\end{array}$ & $\begin{array}{l}\text { bus network optimization through time-dependent } \\
\text { hybrid algorithm }\end{array}$ \\
\hline 10 & Uruguay & 2013 & $\begin{array}{l}\text { Rafael Álvarez Arizpe, Héctor } \\
\text { Martínez luz, Antonio Mauttone }\end{array}$ & $\begin{array}{l}\text { Heurística de búsqueda de entorno variable para el } \\
\text { problema de ruteo de transporte público urbano }\end{array}$ \\
\hline
\end{tabular}

$1^{\text {sh }}$ LACCEI International Multiconference on Entrepreneurship, Innovation and Regional Development - LEIRD 2021: "Ideas to Overcome and Emerge from the Pandemic Crisis", Virtual Edition, December 9-10, 2021. 


\begin{tabular}{|c|c|c|c|c|}
\hline 11 & Uruguay & 2008 & Antonio mauttone; maría e. urquhart & $\begin{array}{l}\text { Una heurística basada en memoria para el problema del } \\
\text { diseño de recorridos en transporte público urbano }\end{array}$ \\
\hline 12 & Uruguay & 2008 & Antonio mauttone, maría e. urquhart & $\begin{array}{l}\text { Optimización multi-objetivo de recorridos y frecuencias } \\
\text { en transporte público aplicado a un caso de estudio real }\end{array}$ \\
\hline 13 & México & 2018 & $\begin{array}{l}\text { Jorge armando estrada Cortez, José } \\
\text { Roberto Pérez cruz, Jaime Saavedra } \\
\text { rosales }\end{array}$ & $\begin{array}{l}\text { Análisis microscópico de los efectos del desorden del } \\
\text { transporte colectivo en el flujo del tránsito urbano }\end{array}$ \\
\hline 14 & Argentina & 2015 & Rodríguez, diego Alejandro & Metaheurística aplicadas a problemas de transporte \\
\hline 15 & Cuba & 2020 & $\begin{array}{l}\text { Ana Camila Pérez Pérez, Eduardo } \\
\text { Sánchez Ansola , Alejandro Rosete } \\
\text { Suárez }\end{array}$ & $\begin{array}{l}\text { Metaheuristics for the solution of the school bus routing } \\
\text { problem }\end{array}$ \\
\hline 16 & Argentina & 2012 & Domínguez, patricia Neri; Cortines & $\begin{array}{l}\text { Un método continuo- discreto para el diseño óptimo de } \\
\text { sistemas de transporte urbano }\end{array}$ \\
\hline 17 & Malasia & 2011 & Joanne suk chun chew; lai soon lee & A genetic algorithm for urban transit routing problem \\
\hline 18 & Chile & 2016 & Javier Vicente Durán Micco & $\begin{array}{l}\text { Optimización en el diseño de redes de transporte público } \\
\text { con objetivos medioambientales mediante algoritmos } \\
\text { evolutivos. }\end{array}$ \\
\hline 19 & España & 2017 & Javier Vásquez peralta & Implementación de un algoritmo de transporte publico \\
\hline 20 & Perú & 2013 & $\begin{array}{l}\text { Eduardo Carbajal López, Lucy } \\
\text { Gabriela Aragón casas, Carmen blancy } \\
\text { Dávila caja huanca }\end{array}$ & $\begin{array}{l}\text { Optimización basada en simulación en un sistema de } \\
\text { tránsito público masivo }\end{array}$ \\
\hline 21 & España & 2014 & $\begin{array}{l}\text { v. corcoba magaña , m. muñoz } \\
\text { organero }\end{array}$ & $\begin{array}{l}\text { Algoritmo para el cálculo de la velocidad media óptima } \\
\text { en una ruta (asga) }\end{array}$ \\
\hline
\end{tabular}

Fuente: Elaboración propia.

Nota. En la tabla 3 se consideran los artículos de investigación científica revisados, el país, el año de publicación, autores, el tema y el resumen general de cada estudio.

Luego de la búsqueda de información con referencia a nuestro tema de investigación "Análisis de métodos algorítmicos para la optimización de rutas en el sistema del transporte urbano" podemos llegar a la conclusión que existe una gran variedad de información muy relevante con nuestro tema de investigación, es por ello que nos centraremos en nuestra pregunta de investigación ¿Cuáles son los métodos o tipos de algoritmos utilizados y/o aplicados a la optimización de transporte urbano en los últimos 12 años?, ante nuestra pregunta de investigación consideramos tomar artículos con 12 años de antigüedad, es decir, con un periodo de año de 2008 2020.

Para poder argumentar nuestra búsqueda de información describiremos los métodos algorítmicos que más se han

aplicado en el sistema de transporte urbano con el objetivó de poder optimizar rutas establecidas y/o crear una nueva ruta.

Como lo explicamos con anterioridad líneas arriba a mediados del siglo pasado, en 1930, se planteó el problema del agente viajero dicho problema, que consiste en obtener la ruta óptima para que un viajero pase por todos los puntos de un mapa, ha sido ampliamente estudiado desde entonces, y su análisis y resolución para encontrar la ruta óptima sigue estando vigente hoy en día [3]. Es por ello por lo que surge una pregunta: ¿Cuál es la ruta más corta posible para llegar a la ciudad? En adelante daremos a conocer ciertas teorías que nos ayuden a absolver esta pregunta.

Algoritmos Genéticos: 
Los algoritmos genéticos son algoritmos evolutivos que se inspiran en el principio de la selección natural y cómo influye la genética en ella. Este tipo de algoritmos simulan la evolución de una población inicial a lo largo de varias generaciones para obtener a los mejores individuos. Por su naturaleza los algoritmos genéticos son adecuados para resolver problemas de optimización complejos y con gran cantidad de factores [3].

Formula de algoritmo Genético:

$$
(i, t)=\sum_{j=1}^{N c}|S(i, j)-c(i, j)|
$$

Donde:

$\mathrm{S}(\mathrm{i}, \mathrm{j})=$ Valor deseado para el individuo i en el caso $\mathrm{j}$

$\mathrm{C}(\mathrm{i}, \mathrm{j})=$ Valor obtenido para por el individuo i para el caso $\mathrm{j}$

$\mathrm{N} \mathrm{c} \quad=$ Número de casos

Según el autor en su artículo de "Optimización de rutas de transporte público" menciona que en la ciudad de Catalunya se presentó un proyecto en donde se debe obtener una optimización de rutas de transporte de una ciudad usando algoritmos genéticos. De esta manera conforme la ciudad valla cambiando con las nuevas necesidades de transporte solo habría que utilizar de nuevo el algoritmo para conseguir la optimización de las rutas existentes o ver la necesidad de crear rutas nuevas.

Como podemos apreciar en el párrafo anterior el autor utilizara este tipo de algoritmo genético, para encontrar la optimización de la ruta, pero además en su artículo nos menciona que esta optimización se lograra con una nueva definición de eficiencia [3], en donde para él, este concepto está amarrado a un factor ecológico. Ya que en este caso de estudio es difícil definir la eficiencia de una línea de transporte y conseguir el equilibrio entre el número de líneas y el número de barrios, es por esto, que el autor crea este nuevo factor. Entonces eso nos quiere decir que, a mayor porcentaje de factor de ecología, menor número de líneas de transporte habrá y también mayor tiempo de espera por parte de los usuarios en los paraderos. Por otro lado, a menor porcentaje de factor de ecología, mayores números de líneas habrá circulado por la vía y esto originará una contaminación (ambiental, visual y sonora) y congestión vehicular, y respecto al tiempo de espera en los paraderos por parte de los usuarios habrá una disminución considerable.

Se llegó a la conclusión que, a mayor porcentaje de ecología, menos optimización se conseguirá y como también a menor porcentaje de ecología mayor optimización se obtendrá, esto requerirá un mayor número de viajes para cubrir la demanda y así obtener resultados óptimos.
Para la implementación del algoritmo genético se puede llevar a cabo atreves de los siguientes lenguajes de programación: Python, dev-c++, Perl o java.

Comparación de Algoritmo de Optimización Tabu Search (TS) y el Algoritmo de Optimización por Enjambre de Partículas (PSO)

El algoritmo tabú (TS) es un procedimiento metaheurístico que se utilizan para resolver problemas de optimización (Glover, 2013). Es posible aceptar soluciones que empeoren la solución actual y la memoria se utiliza para no repetir la trayectoria de la búsqueda, existe dos tipos de memoria: memoria reciente y memoria a largo plazo. En la memoria se guarda atributos de soluciones.

Por otro lado, el algoritmo de optimización por enjambre de partículas (PSO) es un método desarrollado por Kennedy y Eberhart en el año 1995. Este algoritmo define el conjunto de candidatos como un enjambre de partículas que pueden fluir a través del espacio de búsqueda, que son impulsadas por el rendimiento en sí y el mejor rendimiento de sus vecinos.

Según los autores de este artículo "Optimización del transporte público urbano mediante algoritmos de búsqueda Tabú y PSO", en la ciudad de Medellín el sistema del metro tiene un alimentador con la ruta C- 6004 en donde se identificó una serie de problemas como el mayor tiempo de espera en las paradas y largo tiempo de viaje [13]. Estos problemas se han dividido en cuatro aspectos: disponibilidad limitada en la operación del autobús, aumento en las horas extras del conductor, alto consumo de combustible y aumento de kilómetros inefectivos(muertos), aumento de frecuencias, disminución de usuarios e insatisfacción con el servicio (Figura $\mathrm{N}^{\mathrm{a}} 2$ ).

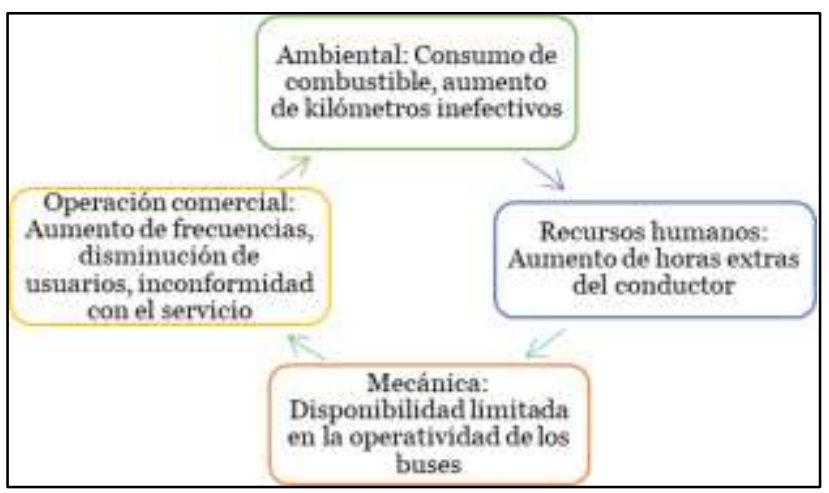

FIGURA N ${ }^{\circ}$ 2: Problemas identificados por departamento [13]

De acuerdo a los problemas mencionados se puede determinar que el proceso más crítico donde se requiere optimizar es en la parte operativa para la mejora del servicio, en consecuencia, el incremento de usuarios y mejora de la frecuencia. 
A razón de ello se aplica y compara los algoritmos (PSO) y (TS) para la realización de la tabla de frecuencias específicamente para la ruta C6-004, perteneciente a las rutas del Sistema Metro de Medellín (30 minutos de viaje). Lo anterior demostró que el sistema debe permitir una mayor flexibilidad con respecto a las paradas que definen la red de la ruta C6-004, además de permitir viajes más directos entre cada par de nodos de origen y destino, que puedan mejorar el nivel de comodidad de los pasajeros, el tiempo de viaje confiable y el ahorro de costos para la empresa prestadora del servicio.

Según los autores nos muestran que para aplicar estos dos tipos de algoritmos tenemos que tener una data establecida en donde se pueda aplicar estos tipos de algoritmos, de acuerdo como muestra la (figura $N^{a} 3$ )

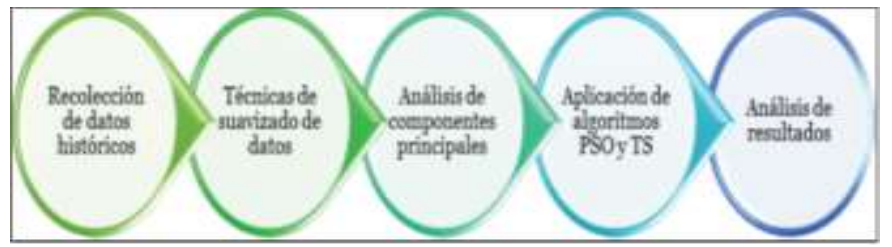

Figura $N^{o} 3$ : Metodología desarrollada [13].

Como vemos los autores buscan reducir el tiempo de viaje por dirección. Una vez obtenido los resultados nos muestra el algoritmo TS que debemos aumentar la velocidad y realizar la parada solo donde hay ascenso o disminución de usuarios, disminuyendo así el tiempo de viaje en 7 minutos. Por otro lado, el algoritmo PSO bajo el mismo escenario logro un ahorro en el tiempo de viaje de 8 minutos y medio. En consecuencia, se opta por el algoritmo TS cumple con el objetivo de la reducción del tiempo de viaje de 30 a 23 minutos, así mismo esto significa un ahorro en horas extras del conductor, reducción de frecuencia, disminución del consumo de combustible y por último al acortarse la frecuencia, la cantidad de pasajeros aumento considerándose así un proceso óptimo para este caso.

\section{Algoritmos de Colonia de Hormigas (ACO)}

El algoritmo de colonia de hormigas atreves de las feromonas emitidas por ellas mismas permiten a las hormigas buscar soluciones de manera conjunta y efectiva, además reduce la probabilidad de quedar atrapado en un punto determinado y mejora la capacidad de búsqueda [14]. Asimismo, este tipo de algoritmos se basa en el estudio de un sistema artificial de hormigas inspiradas en la conducta colectiva de hormigas reales utilizada para resolver problemas de optimización combinatoria. Además, tiene dos objetivos maximizar los recursos encontrados y minimizar los recursos dados [15].

Según los autores de este artículo "Aplicación del Algoritmo de Colonia de Hormigas para la optimización del transporte público", plantean implementar un prototipo que ayude a optimizar el servicio de transporte público de la ciudad de
Guanajuato(México), con el objetivo de brindar un soporte de información o guía, el cual consiste en brindar alternativas óptimas, cantidad de rutas y transportes, tiempos de llegada para dirigirse a su destino, atreves del uso del algoritmo de colonia de hormigas.

Cabe precisar que este tipo de proyectos se puede resolver con la aplicación de algoritmos que incluyen el cumulo de partículas (PSO), algoritmos culturales (ACs) y colonia de hormigas (ACO), debido a que son algoritmos motivados en inteligencia grupal.

La aplicación del algoritmo ACO consta de dos fases; la primera implementa técnicas de búsqueda hacia adelante, la segunda; implementa técnicas de búsqueda hacia atrás. Asimismo, se debe suministrar data de las calles, avenidas de la ciudad, todo ello se procesa bajo el lenguaje de programación JAVA. (Figura 4)

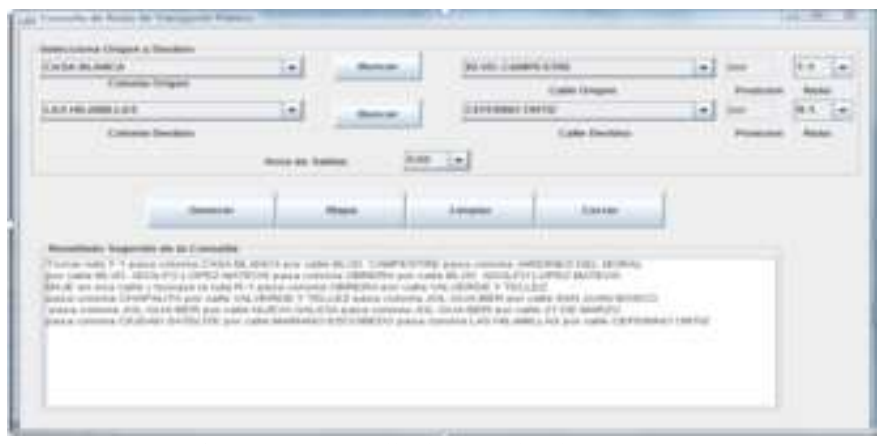

Figura №4: pantalla de consulta de rutas [17].

Es así que permite formar rutas a través de la base de datos con feromonas para su localización dando como resultado un prototipo que procesan las calles como nodos para la aplicación y que esta sigue de cuerdo al algoritmo para formar rutas de transporte que presenta al usuario como recomendaciones. Además, optimiza la búsqueda de la información con lo cual se descarta aquella que no satisface la consulta inicial.

En conclusión, vemos que este prototipo tiene como finalidad que el usuario se transporte de manera eficiente a su destino, este software sirve sobre todo para los que viven en ciudades muy grandes con gran número de rutas de transporte o para usuarios extranjeros que requieran este servicio de transporte, ya que, si no se verán afectados económicamente porque tomaran otro tipo de servicios, además se arriesgan a que le tome más tiempo a su destino.

Cabe precisar que este software puede ser continuamente actualizado ya sea para nuevas calles o rutas. (Figura 5) 


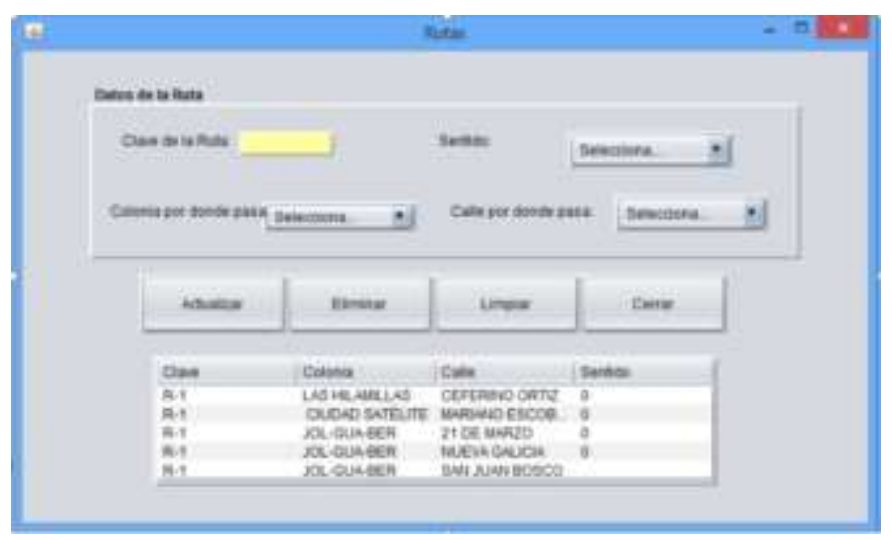

Figura $N^{\circ}$ 5: pantalla para actualizar ruta. (Rodríguez; Guzmán; Hernández; Sánchez; Gutiérrez, 2013)

\section{DISCUSIÓN}

El sistema del transporte urbano en el Perú como se ha venido mencionando en el artículo, es un problema trascendental en nuestro país a lo largo de los años (CAF-Banco de Desarrollo de América Latina, 2011), a razón de ello de acuerdo al análisis se ha identificado distintos métodos algorítmicos como solución para mitigar la problemática en la ruta del sistema de transporte.

El presente artículo analiza tres casos: Algoritmo genético, algoritmo Tabu Search (TS) - el algoritmo por enjambre de partículas (PSO) y algoritmos de colonia de hormigas (ACO).

El algoritmo genético es aplicado al sistema de transporte urbano, considerando un factor ecológico para la optimización del mismo, en donde a mayor factor ecológico (100\%), menor cantidad de líneas habrá y un aumento de tiempo de espera en los paraderos por parte de los usuarios y por ello menor será la optimización de la ruta [3]. A razón de ello se recomienda que este factor sea intermedio (50\%) para lograr un equilibrio y una optimización de la ruta. Este método se podría decir que se aplicó en Lima- Perú en el gobierno municipal en el periodo 2011-2014 (Municipalidad de Lima 2020) en la ruta Tacna Garcilaso - Arequipa (corredor azul) [24], en donde se priorizo este factor ecológico desplazando o retirando a las unidades de distintas líneas convencionales y dejando únicamente operar al corredor azul trayendo como consecuencia una sobredemanda y la aparición del transporte informal(taxi colectivo) por lo que no se recomienda este método algorítmico en Lima.

En cuanto al algoritmo Tabú Search (TS) y PSO nos menciona que la aplicación de estos algoritmos nos ayuda a reforzar la parte operativa en donde nos permita minimizar tiempos de viaje considerando las paradas innecesarias en los paraderos, asimismo esto trae como consecuencia el ahorro en el costo de combustible, en el costo de planilla por horas extras y se optimiza el tiempo de viaje [13]. Este método algorítmico es recomendable implementarlo al sistema de transporte de Lima, ya que si se aplica este método en las empresas de transporte convencional no solo se obtendría ahorros o disminución de los gastos operativos ( horas extra, combustible y otro), si no que a nivel macro el hecho de minimizar el tiempo de viaje contribuye a un descongestionamiento vehicular puesto que las unidades optimizarían el tiempo, realizando paradas donde solamente aborden o desciendan del buz, en caso no haya esta acción el buz continuara su recorrido lo cual es recomendable su aplicación.

Por último, el autor propone la aplicación del algoritmo colonia de hormigas en donde permite al usuario tener distintas alternativas de ruta para dirigirse a su destino optimizando así su tiempo [17]. Este método algorítmico en el Perú ha sido implementado a través de un aplicativo llamado Tu Ruta, en la ciudad de Lima, el cual muestra al usuario que líneas de transporte abordar, en que paradero esperar, en que paradero bajar, permitiendo llegar a los usuarios a destinos desconocidos en un menor tiempo. Este aplicativo es eficiente y viable para contribuir en solución al problema señalado sin embargo existen muchas empresas de transporte convencional que aún no son parte de este aplicativo, por lo cual se recomienda que para una mayor eficiencia sean participe de este esté aplicativo. Además para dar mayor impacto en la solución se podría implementar al aplicativo una opción en donde permita al usuario enviar una señal, indicando que se requiere el servicio y asimismo el conductor de la unidad pueda visualizar que en el próximo paradero hay usuarios que quieran abordar dicha unidad, caso contrario no se envié la señal de requerimiento del servicio el mismo deberá continuar su recorrido sin realizar parada alguna, generando así disminución en el tiempo de viaje de la unidad y un descongestionamiento a nivel general de la ruta.

\section{CONCLUSIONES:}

Todos somos conscientes de la gran problemática que estamos viviendo las grandes ciudades de Latinoamérica y el mundo respecto al sistema de transporte, y Lima no es ajeno a este problema, el cual se busca encontrar múltiples soluciones con distintos métodos para optimizarlo en tiempo y en forma para darle mejor calidad de vida a nuestros habitantes.

Implementar un algoritmo genético nos da un panorama eficiente ante problemas de optimización en escenarios reales que contienen la mejor configuración de las líneas de transporte, asimismo para implementarlo se hace difícil definir la eficiencia de una línea de transporte para desarrollar un equilibro entre el número de líneas y barrios, ya que el factor de ecología cuando es máximo se va a ver influenciado en la optimización, es decir, cuanto mayor es la factor ecológico menor optimización habrá y cuando menor es el factor ecológico mayor optimización se conseguirá, es por ende que no se recomienda aplicarlo a la ciudad de Lima por la cantidad de habitantes que se movilizan a lo largo del día, considerar la ecología significaría quitar las rutas convencionales y esto generaría largas colas de espera en los paraderos y la aparición del transporte informal. 
En cuanto al algoritmo Tabú y PSO se obtuvieron resultados satisfactorios porque ambos cumplen con el objetivo de optimizar el tiempo de viaje y reducir la frecuencia entre vehículos.

Por lo tanto, se recomienda utilizar el algoritmo Tabu, porque logro una optimización en tiempo más óptimo que el algoritmo PSO, por lo que ayudara a resolver los problemas de tráfico y tiempo de viaje que perjudica al sistema de transporte de Lima, porque adicionalmente aplicar este algoritmo refuerza la parte operativa trayendo como consecuencia el ahorro en el costo de combustible y el ahorro en el costo de horas extras en las planillas de operadores de buses. Esto genera mayor margen de ganancias tanto para las empresas de transporte y para los usuarios en tiempo de viaje, por lo que se realizara paradas solo donde haya usuarios que abordaran el bus permitiendo minimizar el tiempo de llegada a los destinos de cada usuario.

Por último, el algoritmo de Colonia de Hormigas nos permite optimizar un conjunto de rutas para el usuario en función de origen y destino, por ende, al aplicarlo o implementarlo no nos estaríamos centrando en el problema macro que es el sistema de trasporte en general, sino en un solo sector que es el transporte particular o individual por lo que no se recomienda aplicarlo.

\section{REFERENCIAS}

[1] Gestal M, Rivero D, Rabuñal J, Dorado J y Pazos A. (2010) Introducción a los Algoritmos Genéticos y la Programación Genética. Universidad de Coruña, Madrid.

[2] López J. (2009) Algoritmos y Programación (Guía para Docentes). Recuperado https://libros.metabiblioteca.org/bitstream/001/169/8/AlgoritmosProgra macion.pdf

[3] Kanaan S, Ventura C. (2016) Optimización de rutas de transporte público con Algoritmos Genéticos. Recuperado de http://openaccess.uoc.edu/webapps/o2/bitstream/10609/59085/7/mgeno vardTFM1216memoria.pdf

[4] INEI. (2018). Censos Nacionales 2017: XII de Población, VII de Vivienda y III de Comunidades Indígenas. Recuperado de: https://www.inei.gob.pe/media/MenuRecursivo/publicaciones_digitales/ Est/Lib1539/libro.pdf

[5] Neyra L, Ingunza H. (2019). "La calidad del servicio del corredor azul y su relación con la satisfacción de sus usuarios de la avenida Arequipa en el 2019". Recuperado de https://repositorio.utp.edu.pe/bitstream/handle/20.500.12867/3271/Luis $\% 20$ Neyra_Herly\%20Ingunza_Trabajo\%20de\%20Investigacion_Bachil ler_2019.pdf?sequence $=1 \&$ isAllowed $=y$

[6] Barbero J. (2006) TRANSPORTE URBANO. En Perú La oportunidad de un país diferente Prospero, equitativo y gobernable. Recuperado de: https://www.mef.gob.pe/contenidos/pol_econ/documentos/BM_Peru_un _pais_diferente.pdf

[7] Romero Pastor, L; López Valdez, B; Vargas La Serna, J; Reátegui Del Águila, L; de La Barrera Laca, L. (2019). Movilidad Urbana en Lima y Callao, Caso de Estudio: La Autoridad de Transporte Urbano para Lima y Callao - ATU. Recuperado de: https://repositorioacademico.upc.edu.pe/bitstream/handle/10757/631318 /Romero_PL.pdf?sequence=3\&isAllowed $=\mathrm{y}$

[8] Espíritu N. (2018) El Transporte y el Estrés en la Ciudad de Lima. Recuperado https://repositorio.urp.edu.pe/bitstream/handle/URP/1486/8.\%20Espirit u $\% 20$ Salinas $\% 20$ Natividad $\% 2 \mathrm{C} \% 20 \mathrm{E} 1 \% 20$ transporte $\% 20 \mathrm{y} \% 20 \mathrm{el} \% 20 \mathrm{e}$ str\%C3\%A9s\%20enla\%20ciudad \%20de \%20Lima.pdf?sequence=1\&is Allowed=y

[9] MTC. (2019). Plan Estratégico Institucional PEI 2018-2022. Recuperado de: $\quad$ http://portal.mtc.gob.pe/nosotros/documentos/pei/PEI(2018 2022).pdf

[10] Muñoz D. (2014). programación del Horario de Salidas y asignación de Buses para un Alimentador del Transantiago. Recuperado de: http://repositorio.uchile.cl/handle/2250/130306

[11] Beltrán G., Óscar A. (2005). Revisiones sistemáticas de la literatura. Recuperado de: https://www.redalyc.org/pdf/3377/337729264009.pdf

[12] Glover F. (2013). TABU SEARCH: Effective Strategies for Hard Problems in Analytics and Computational Science. Recuperado de: http://leeds-

faculty.colorado.edu/glover/449\%20-\%20Tabu\%20Search\%20in\%20A nalytics\%20and\%20Computational\%20Science.pdf

[13] Betancourt L, Becerra M. (2019). Optimización del Transporte Público Urbano Mediante Algoritmos de Búsqueda Tabú y PSO: Medellín, Colombia. Recuperado de: https://search.proquest.com/openview/ec8601c82489c20f58286629e316 c348/1 ?pq-origsite $=$ gscholar $\&$ cbl $=1006393$

[14] Min X, Zhang J, Li Y. (2008). Orthogonal Methods Based Ant Colony Search for Solving Continuous Optimization Problems. Recuperado de: http://eprints.gla.ac.uk/3894/1/JCST_paper.pdf

[15] Gómez C; Cruz L; Meza E; Amaro C; Aguirre M; Ochoa C. (2009). Análisis de rendimiento del algoritmo de búsqueda de hormigas vecinas a través del diseño del experimento. Recuperado de https://link.springer.com/chapter/10.1007/978-3-642-02319-4_80

[16] CAF. (2011). Desarrollo urbano y movilidad en América Latina. Caracas: CAF. Recuperado de: http/handle/123456789/419://scioteca.caf.com

[17] Rodríguez C. (2011). Aplicación del Algoritmo de Colonia de Hormigas para la Optimización del Transporte Público. Recuperado de: http://www.itcelaya.edu.mx/ojs/index.php/pistas/article/view/1266

[18] Domingueza P, Cortínez V. (2012). Un Método Continuo-Discreto para el Diseño Óptimo de Sistemas de Transporte Urbano. Recuperado de: https://ri.conicet.gov.ar/bitstream/handle/11336/79483/CONICET_Digit al_Nro.7b812ccf-7790-4a94-b2c40a6c22e6d02e_A.pdf?sequence $=2$ \&isAllowed $=y$

[19] Durán J. (2016). Optimización en el Diseño de Redes de Transporte Público con Objetivos Medioambientales Mediante Algoritmos Evolutivos. Recuperado de: http://152.74.17.92/bitstream/11594/1938/1/Tesis_Optimizacion_en_el_ dise\%c3\%b1o_de_redes_de_Transporte.Marked.pdf

[20] Corcoba V, Muñoz M. (2014). Algoritmo para el cálculo de la velocidad media óptima en una ruta (ASGA). Recuperado de: file:///C:/Users/user/Downloads/9436-34284-1-SM\%20(1).pdf

[21] López J. (2011). Enfoque Multiobjetivo de una Metaheurística Híbrida Para la Optimización de Rotaciones de Transporte Terrestre de Pasajeros como Base de un Sistema de Soporte de Decisiones Empresarial Recuperado http://laccei.org/LACCEI2011Medellin/RefereedPapers/LO311_Lopez. pdf

[22] Carbajal E, Aragón L, Dávila C. (2013). Optimización Basada en Simulación en un Sistema de Tránsito Público Masivo. Recuperado de: http://www.laccei.org/LACCEI2013Cancun/RefereedPapers/RP110.pdf

[23] Mauttone A, Urquhart M. (2008). Optimización Multi-Objetivo de Recorridos y Frecuencias en Transporte Público Aplicado a un Caso de Estudio Real. Recuperado de: https://sochitran.cl/static/upload/archivos/sochitran/4186/14/146135195 8.pdf

[24] Fowks J, (2015). La reforma del transporte de Lima, en la incertidumbre. Recuperado https://elpais.com/internacional/2015/01/02/actualidad/1420209045_43 6816.html 